Boise State University

ScholarWorks

$11-2012$

\title{
A Modified Delay-Time Method for Statics Estimation with the Virtual Refraction
}

\author{
T. Dylan Mikesell \\ Boise State University \\ Kasper van Wijk \\ Boise State University \\ Elmer Ruigrok \\ Delft University of Technology \\ Andrew Lamb \\ Boise State University \\ Thomas E. Blum \\ Boise State University
}




\title{
A modified delay-time method for statics estimation with the virtual refraction
}

\author{
T. Dylan Mikesell ${ }^{1}$, Kasper van Wijk ${ }^{2}$, Elmer Ruigrok ${ }^{3}$, Andrew Lamb $^{2}$, and Thomas E. Blum²
}

\begin{abstract}
Topography and near-surface heterogeneities lead to traveltime perturbations in surface land-seismic experiments. Usually, these perturbations are estimated and removed prior to further processing of the data. A common technique to estimate these perturbations is the delay-time method. We have developed the "modified delay-time method," wherein we isolate the arrival times of the virtual refraction and estimate receiver-side delay times. The virtual refraction is a spurious arrival found in wavefields estimated by seismic interferometry. The new method removes the source term from the delay-time equation, is more robust in the presence of noise, and extends the lateral aperture compared to the conventional delay-time method. We tested this in an elastic 2D numerical example, where we estimated the receiver delay-times above a horizontal refractor. Taking advantage of reciprocity of the wave equation and rearranging the common shot gathers into common receiver gathers, isolated source delay times could also be obtained.
\end{abstract}

methods based on refraction analysis are often used to estimate source and receiver statics. Section 3.4 of Yilmaz (2001) provides a background on refraction statics corrections. One such refraction method is the delay-time (DT) method (e.g., p. 120 in Burger et al., 2006) which estimates individual source and receiver statics, assuming that the refractor has a shallow dip $\left(<10^{\circ}\right)$.

Here we introduce a new technique for statics estimation that uses arrival times of the virtual refraction artifact (Dong et al., 2006; Mikesell et al., 2009). This artifact is a result of applying crosscorrelation-type seismic interferometry to data containing refractions. In this method, we crosscorrelate the wavefields recorded at two receivers, $X_{A}$ and $X_{B}$, to estimate the Green's function between the two receivers (Curtis et al., 2006; Wapenaar and Fokkema, 2006). Crosscorrelating multiple receivers with $X_{A}$, and summing correlations from many sources, we create a virtual shot record having a virtual source at $X_{A}$. By definition, the virtual refraction is the first arrival in the virtual shot record (Mikesell et al., 2009). This characteristic, and its robustness in the presence of noise due to inherent summing over many seismic sources, make it ideal to calculate refraction statics. After a brief overview of the delay-time method, we develop a modified delay-time (MDT) method and highlight its benefits with a noisy $2 \mathrm{D}$ numerical experiment.

\section{THE DELAY-TIME METHOD}

\section{INTRODUCTION}

Shallow lateral velocity heterogeneity and topography variations are known to cause traveltime perturbations in the recorded seismic wavefield. These distortions negatively affect normal moveout velocity analysis (e.g., Figures 3.0-3.6 in Yilmaz, 2001), resulting in a suboptimal seismic image. Such traveltime perturbations, referred to as field statics, need to be removed early in the reflection seismic imaging process (e.g., Figures 3.3-3.12 in Yilmaz, 2001). When the depth and velocity of the weathering layer are poorly known, In the DT method (Burger et al., 2006), the refraction traveltime equation is

$$
T_{S_{i} X_{j}}=d T_{S_{i}}+d T_{X_{j}}+\frac{\left|S_{i}-X_{j}\right|}{V_{2}},
$$

where $\left|S_{i}-X_{j}\right|$ is the horizontal distance between a source $\left(S_{i}\right)$ and a receiver $\left(X_{j}\right), V_{2}$ is the refractor velocity, and $d T_{S_{i}}$ and $d T_{X_{j}}$ are delays associated with propagation through the near surface for each source and receiver, respectively (Figure 1). The delay-time is defined as

\footnotetext{
Manuscript received by the Editor 3 April 2012; revised manuscript received 24 July 2012; published online 16 October 2012.

${ }^{1}$ Géoazur, Observatoire de la Côte d'Azur, Université de Nice Sophia-Antipolis, Valbonne, France and Boise State University, Physical Acoustics Laboratory, Department of Geosciences, Boise, Idaho, USA. E-mail: dmikesell@cgiss.boisestate.edu.

${ }^{2}$ Boise State University, Physical Acoustics Laboratory, Department of Geosciences, Boise, Idaho, USA. E-mail: kaspervanwijk@boisestate.edu; andrewlamb@u.boisestate.edu; tblum@cgiss.boisestate.edu.

${ }^{3}$ Delft University of Technology, Department of Geoscience \& Engineering, Delft, The Netherlands. E-mail: e.n.ruigrok@tudelft.nl.

(c) 2012 Society of Exploration Geophysicists. All rights reserved.
} 


$$
d T=\frac{Z \cos \theta}{V_{1}}
$$

where $Z$ is the local weathering layer thickness, $V_{1}$ is the local velocity, and $\theta$ is the critical angle. For one source $(i=1)$ and multiple receivers $(j=1, \ldots, k)$, equation 1 can be written as a system of equations, $d=A m$, where

$$
d=\left[T_{S_{1} X_{1}}, T_{S_{1} X_{2}}, \ldots, T_{S_{1} X_{k}}\right]^{T}
$$

and

$$
m=\left[d T_{S_{1}}, d T_{X_{1}}, d T_{X_{2}}, \ldots, d T_{X_{k}}, 1 / V_{2}\right]^{T} .
$$

In this notation, []$^{T}$ represents the vector transpose. The linear operator,

$$
\mathbf{A}=\left(\begin{array}{ccc}
1 & & \left|S_{1}-X_{1}\right| \\
\vdots & \left(I_{k \times k}\right) & \vdots \\
1 & & \left|S_{1}-X_{k}\right|
\end{array}\right),
$$

which is a horizontal concatenation of two column vectors and an identity matrix. The first column is related to the source delay time and the last column is associated with the last term $\left|S_{i}-X_{j}\right| / V_{2}$ in equation 1 . We can invert this system of equations (described later) to estimate $\mathbf{m}$ from refraction first-break picks. Because this method is based on the refracted wave only, before inversion we must remove all first-break picks associated with other wave modes. In field data applications, the user visually inspects the picks. The picks corresponding to other modes (e.g., the direct-P arrival) are eliminated before inversion. To extend the results for $n$ sources, we vertically concatenate the data from each new source, as well as model parameters. As a result, $\mathbf{A}$ is augmented on the left with columns of zeros related to each source position, so that the size of $\mathbf{A}$ is $(n \times k) \times(n+k+1)$.

\section{THE MODIFIED DELAY-TIME METHOD}

An underlying principle in seismic interferometry is that the common paths (e.g., the direct waves in Bakulin and Calvert, 2006) of waves traveling to two receivers are removed. Crosscorrelating the refraction arrivals from $S_{1}$ at receivers $X_{1}$ and $X_{2}$ (Figure 1) eliminates common paths in the two arrival-time relations $T_{S_{1} X_{1}}$ and $T_{S_{1} X_{2}}$. The paths that cancel are (1) from source $S_{1}$ to the interface $d T_{S_{1}}$, (2) the path along the interface up to the dashed-blue line, and (3) from the interface to receiver $X_{1}: d T_{X_{1}}$. The paths that are not in common are (1) the virtual refraction path (solid-green arrow), and (2) the path difference through the weathering layer that the

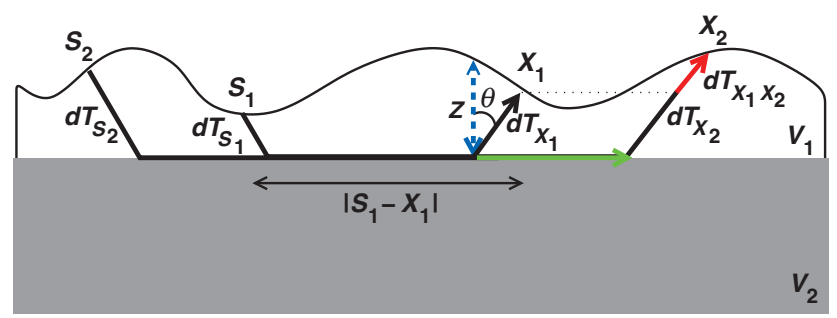

Figure 1. A model with a laterally varying weathering layer thickness with sources $S_{i}$ and receivers $X_{j}$. Various traveltimes along the refraction path are indicated. See text for details. refraction travels to $X_{2}$ compared to $X_{1}$ (solid-red arrow). For a horizontal refractor and any source $i$ that generates a refraction at both receivers, crosscorrelation results in an arrival-time difference equation

$$
T_{X_{1} X_{2}}=T_{S_{i} X_{2}}-T_{S_{i} X_{1}}=d T_{X_{1} X_{2}}+\frac{\left|X_{1}-X_{2}\right|}{V_{2}} .
$$

The right hand side of equation 3 represents the virtual refraction $\left(\left|X_{1}-X_{2}\right| / V_{2}\right)$, plus the traveltime perturbation $\left(d T_{X_{1} X_{2}}=\right.$ $d T_{X_{2}}-d T_{X_{1}}$ ) between the two receivers. The virtual refraction has two important properties. First, it is by definition the first arrival in the virtual shot record if the correlated wavefields contain refractions from the same interface. This property relies only on the assumption that no out-of-plane diffractors contribute significant energy to the real 2D shot records. Second, the virtual refraction is the result of a summation of sources. This means that the virtual refraction has a larger $\mathrm{S} / \mathrm{N}$ than the real refraction (Mallinson et al., 2011). These properties make the virtual refraction artifact a useful arrival for refraction statics estimation. The perturbation $d T_{X_{1} X_{2}}$ is the receiver static at $X_{2}$ relative to the reference receiver $X_{1}$. A similar relationship for core-mantle diffractions is given in Ruigrok et al. (2012).

Similar to the DT method, equation 3 can be written as a system of equations, $d=A m$, for one source and $k$ receivers where

$$
\begin{aligned}
d & =\left[T_{X_{1} X_{1}}, T_{X_{1} X_{2}}, \ldots, T_{X_{1} X_{k}}\right]^{T}, \\
m & =\left[d T_{X_{1} X_{1}}, d T_{X_{1} X_{2}}, \ldots, d T_{X_{1} X_{k}}, 1 / V_{2}\right]^{T},
\end{aligned}
$$

and

$$
\mathbf{A}=\left(\begin{array}{cc} 
& \left|X_{1}-X_{1}\right| \\
\left(I_{k \times k}\right) & \vdots \\
& \left|X_{1}-X_{k}\right|
\end{array}\right) .
$$

Elements of the last column in $\mathbf{A}$ are the distances between the receivers and the virtual source (at $X_{1}$ ). To incorporate more virtual source locations, we vertically concatenate this system of equations for each virtual source. Note that A now lacks the column related to the source term. This has been removed by the crosscorrelation operator. Furthermore, the receiver terms are estimated relative to each virtual source position (e.g., $X_{1}$ in the above example).

\section{THE INVERSE PROBLEM}

In either the DT or MDT method, delay times $\mathbf{m}$ can be estimated from real or virtual refraction arrival times $\mathbf{d}$ and used for statics corrections. There are many approaches to the inverse problem. We use a truncated singular-value decomposition (e.g., p. 55 in Aster et al., 2005) to estimate the pseudoinverse operator $A^{\dagger}$ such that

$$
m^{\dagger}=A^{\dagger} d,
$$

where $\mathrm{m}^{\dagger}$ represents the truncated solution based on a singularvalue tolerance. In this case, the tolerance is computed as $(\max (\operatorname{size}(\mathbf{A})) *\|\mathbf{A}\| \epsilon)$, where $\epsilon=2.22 e^{-16}$ and $\|\mathbf{A}\|$ is the norm of the matrix A. 


\section{AN ELASTIC 2D NUMERICAL EXAMPLE}

We compare the DT and MDT methods with a noisy elastic wave 2D numerical experiment. The model is shown in Figure 2a. Under the left half of the receiver array (receiver positions 500-1000 m), the surface layer thickness is constant at $100 \mathrm{~m}$. Under the right half (receiver positions 1000-1500 m), the surface layer thickness varies sinusoidally, leading to a layer thickness that varies between 90 and $110 \mathrm{~m}$. The model velocities and densities given in Figure 2a are constant in each layer. We use the spectral element method (Komatitsch and Vilotte, 1998; Komatitsch and Tromp, 2002) to model $0.5 \mathrm{~s}$ of the vertical component seismic wavefield at each receiver (green triangles) from a vertical point force (blue stars). The source is a $40 \mathrm{~Hz}$ Ricker wavelet.

We show the wavefield recorded from a source at $500 \mathrm{~m}$ in Figure 2b. Strong hyperbolic moveout reflections, as well as linear moveout Rayleigh, direct $\mathrm{P}$, and refracted waves are visible. Surface-layer thickness variations result in short-wavelength fluctuations in the refracted wave and far-offset reflections. First-break arrival times are picked using the modified-energy ratio (abbreviated MER; see Han et al., 2008) and are denoted in red. We also pick the times of the first arriving wave for a source positioned at $1500 \mathrm{~m}$ to have data in the reverse direction. We chose to use the MER method because it more accurately picks first break in the presence of uncorrelated noise. Han et al. (2010) demonstrate this in a comparison with the short-term average to long-term-average ratio method of Earle and Shearer (1994).

To demonstrate the robustness of the virtual refraction compared to the real refraction, we add enough Gaussian white noise to the numerical data so that the automated MER picker starts to fail at far offsets (Figure 2b). Before creating the virtual shot record, we suppress other arrivals in the real shot records. By muting the wavefield $25 \mathrm{~ms}$ after the direct and refracted arrivals, we eliminate correlations of arrivals other than the direct or refracted waves. We create virtual shot records by crosscorrelating the wavefields at the red star receivers in Figure 2a with all other receivers in the array. The virtual shot at $800 \mathrm{~m}$ gives the forward profile virtual refraction and the virtual shot at $1200 \mathrm{~m}$ gives the reverse profile. We sum crosscorrelations from 10 evenly spaced sources (e.g., from 500 to $545 \mathrm{~m}$ for the virtual shot at $800 \mathrm{~m}$ ). These sources are positioned past the critical offset from the virtual shot and all receivers to the right. Thus, the virtual refraction signal-to-noise ratio is increased by $\sim \sqrt{N}$, where $N$ is the number of sources in the summation (Mikesell et al., 2009; Bharadwaj et al., 2011; Mallinson et al., 2011). The virtual shot at $800 \mathrm{~m}$ is shown in Figure 2c, where the first break is now the phase that goes through $t=0 \mathrm{~s}$ at the virtual shot position. These arrival time picks are again shown in red. The increase in $\mathrm{S} / \mathrm{N}$ improves the accuracy of the picks in Figure 2c compared to $2 \mathrm{~b}$.

Using these noisy refraction arrival time data, we compare the receiver delay-time estimates $\left(\mathrm{m}^{\dagger}\right)$ from the MDT method (red line) with those from the DT method (blue line) in Figure 3. In both cases, the first-break data in the forward and reverse directions are combined and smoothed before inversion. We use a three-point convolutional smoothing operation. Without smoothing, the DT result is too noisy to compare with the MDT result. We apply smoothing to the virtual refraction first breaks so we can make an equal comparison, even though the MDT result is stable by itself.

The DT and MDT methods estimate the refractor velocity with similar accuracy, which is influenced by the quality of the
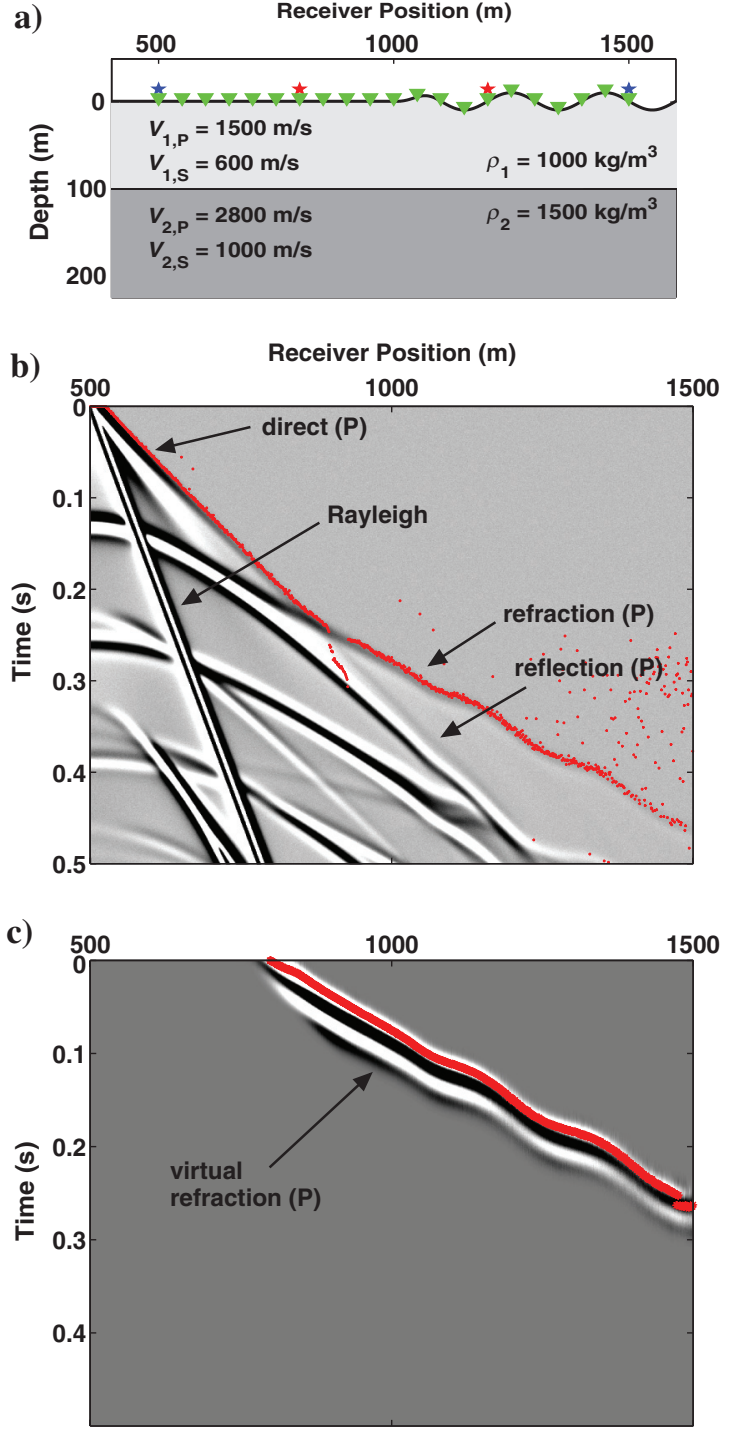

Figure 2. (a) Elastic model with varying surface layer thickness. Blue stars are real sources and red stars are virtual sources. Green triangles are receivers located at the surface. Velocities and densities are constant in each layer. (b) Shot record and first-break picks for a real source at $500 \mathrm{~m}$ after adding Gaussian white noise. (c) Virtual shot record for a virtual source at $800 \mathrm{~m}$.

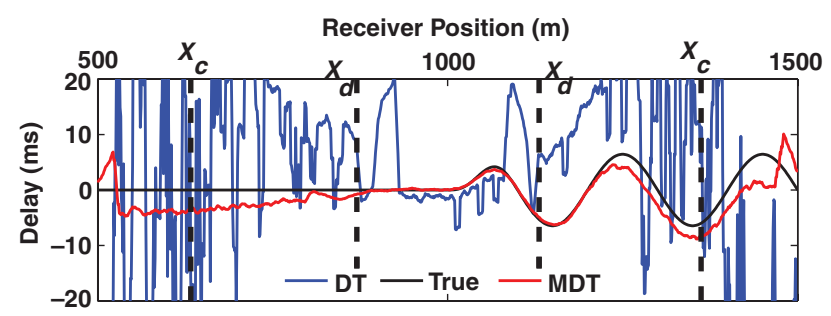

Figure 3. The MDT method receiver delay times (red) and DT method (blue). The true receiver delay times relative to zero elevation are represented by the thin black line. The thick dashed black lines indicate the crossover $\left(X_{d}\right)$ and critical-offset $\left(X_{c}\right)$ distances for forward and reverse shots. 
first-break picks and the geometry of the source and receiver arrays. The influence of the noise on our first-break picks is apparent in the delay-time estimates shown in Figure 3. The virtual refraction picks are more accurate at farther offsets, resulting in superior delay-time estimates. The inherent stacking over sources in the virtual shot generation creates a higher $\mathrm{S} / \mathrm{N}$ in the virtual shot record, which leads to more accurate picks of the virtual refraction.

\section{DISCUSSION}

\section{Lateral aperture}

The DT and MDT methods rely on large offset data containing refraction first breaks; however, MDT offers an increased lateral aperture compared with the DT method. We illustrate this by considering the traveltimes of the coherent events in the real and virtual shot records. Figure 4a shows the arrival times of the direct (black), reflected (red) and refracted (blue) waves for real sources at both ends of the receiver array. The crossover distance $\left(X_{d}\right)$ indicates where the real refraction becomes the first arrival. In the DT method, we use arrival times from distances past $X_{d}$ from each source and we eliminate data from distance less than $X_{d}$.

a)

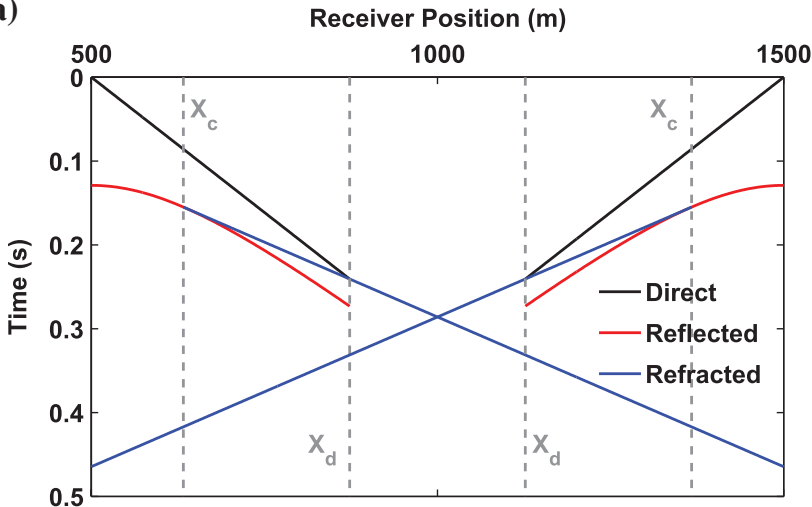

b)

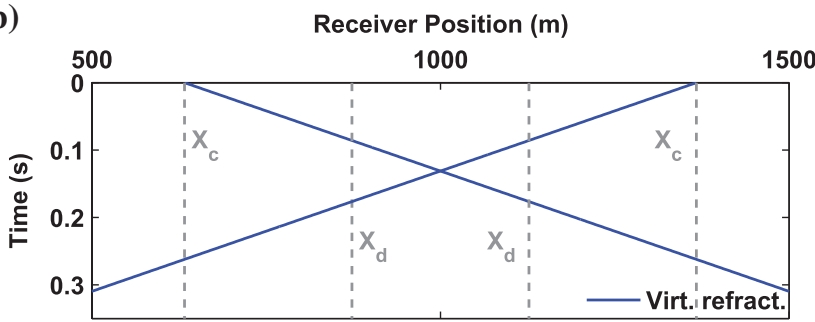

c)

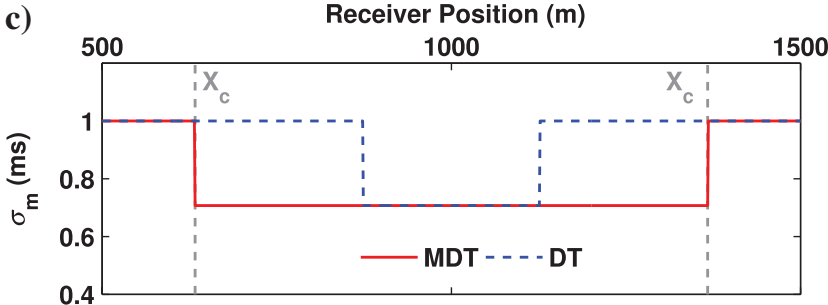

Figure 4. (a) Traveltimes of three wave types for sources at the edges of the receiver array. (b) Traveltimes of the virtual refraction. (c) Comparison of the model parameter standard deviation $\left(\sigma_{m}\right)$ for each receiver.
In contrast, for any virtual shot location past the critical offset $\left(X_{c}\right)$ from the real source, the virtual refraction is the first arrival. Figure $4 \mathrm{~b}$ shows the arrival times of the virtual refractions for virtual sources at $X_{c}$ from each real source in Figure 4a. From Figure 4a and $4 \mathrm{~b}$, we conclude that we can extend our range of usable firstarrival data from $X_{d}$ to $X_{c}$ with the virtual refraction. Tatanova et al. (2009) investigate a similar increase in lateral aperture using the virtual refraction for time-lapse reservoir characterization. Moreover, to determine the $X_{c}$ distance and appropriate virtual shot positions, the semblance method presented in Mikesell and van Wijk (2011) could be employed.

In either the DT or MDT method, the error in receiver delay-time is smallest within sections of the model where forward and reverse propagating (real or virtual) refractions are the first arrival. We can quantify the error reduction by calculating the diagonal of the model covariance matrix estimated from the matrix $\mathbf{A}$ given in equation 2 or equation 4 :

$$
\sigma_{m}^{2}=\sigma_{d}^{2} *\left(\mathbf{A}^{T} \mathbf{A}\right)^{-1}
$$

Assuming a constant data variance $\left(\sigma_{d}^{2}=1 \mathrm{~ms}\right)$, we establish a relative delay-time error $\left(\sigma_{m}\right)$ for each receiver. Figure $4 \mathrm{c}$ shows $\sigma_{m}$ for the DT (dashed blue) and MDT (solid red) methods using the source and receiver geometry in the 2D numerical experiment. This example illustrates that the MDT has reduced delay-time error over a larger lateral extent compared to the DT method. This is a direct result of the virtual refraction being the first arrival over a larger region of the model (Figure $4 \mathrm{a}$ and $4 \mathrm{~b}$ ).

\section{Source delay time}

In the MDT method, the source delay time is eliminated from the inversion process. To estimate the source delay time, we can rearrange the data into common receiver gathers (rather than common source gathers) and repeat the same steps outlined above. Curtis et al. (2009) demonstrate how to turn sources into virtual receivers using the reciprocity of the wave equation and seismic interferometry. Following this, a modified source delay-time equation is

$$
T_{S_{2} X_{j}}-T_{S_{1} X_{j}}=d T_{S_{1} S_{2}}+\frac{\left|S_{1}-S_{2}\right|}{V_{2}},
$$

where $d T_{S_{1} S_{2}}$ is now a source delay time relative to $S_{1}$. Therefore, we have developed a novel method that isolates the two types of delay times. This approach eliminates any trade-off that may exist between the source and receiver delay times in the traditional DT method.

\section{CONCLUSIONS}

We have presented the modified delay-time method for statics estimation based on the virtual refraction. An elastic numerical example illustrates how to isolate source and receiver delay times before inversion, thus removing any trade-off that exists within the delay-time method. Because we stack over many sources in the seismic interferometry processing, the virtual refraction arrival time picks are more robust in the presence of random noise. In addition, we gain an increased lateral aperture by using the virtual refraction. The result is a more accurate delay time estimate for statics corrections compared to the traditional delay-time method. 


\section{ACKNOWLEDGMENTS}

D.M. was supported by an NSF GK-12 Graduate Fellowship and ION Geophysical during this work. K.W. was supported by the NSF grant EAR-1142154. We thank Matt M. Haney for the convolutional smoothing code as well as Simon King, and Kevin Bishop for constructive reviews that improved the quality of this paper.

\section{REFERENCES}

Aster, R. C., B. Borchers, and C. H. Thurber, 2005, Parameter estimation and inverse problems: Elsevier.

Bakulin, A., and R. Calvert, 2006, The virtual source method: Theory and case study: Geophysics, 71, no. 4, SI139-SI150, doi: 10.1190/1 .2216190 .

Bharadwaj, P., G. Schuster, I. Mallinson, and W. Dai, 2012, Theory of supervirtual refraction interferometry: Geophysical Journal International, 188 263-273, doi: 10.1111/gji.2012.188.issue-1.

Burger, H. R., A. F. Sheehan, and C. H. Jones, 2006, Introduction to applied geophysics: Exploring the shallow subsurface: W. W. Norton \& Company.

Curtis, A., P. Gerstoft, H. Sato, R. Snieder, and K. Wapenaar, 2006, Seismic interferometry - Turning noise into signal: The Leading Edge, 25, 10821092, doi: 10.1190/1.2349814.

Curtis, A., H. Nicolson, D. Halliday, J. Trampert, and B. Baptie, 2009, Virtual seismometers in the subsurface of the earth from seismic interferometry: Nature Geoscience, 2, 700-704, doi: 10.1038/ngeo615.

Dong, S., J. Sheng, and J. T. Schuster, 2006, Theory and practice of refraction interferometry: 76th Annual International Meeting, SEG, Expanded Abstracts, 3021-3025.
Earle, P. S., and P. M. Shearer, 1994, Characterization of global seismograms using an automatic-picking algorithm: Bulletin of the Seismological Society of America, 84, 366-376.

Han, L., J. Wong, and J. C. Bancroft, 2010, Time picking on noisy microseismograms: GeoCanada Meeting.

Han, L., J. Wong, J. C. Bancroft, and R. R. Stewart, 2008, Automatic time picking and velocity determination on full waveform sonic well logs: Technical Report 20, CREWES, University of Calgary.

Komatitsch, D., and J. Tromp, 2002, Spectral-element simulations of global seismic wave propagation - I. Validation: Geophysical Journal International, 149, 390-412, doi: 10.1046/j.1365-246X.2002.01653.x.

Komatitsch, D., and J.-P. Vilotte, 1998, The spectral element method: An efficient tool to simulate the seismic response of 2D and 3D geological structures: Bulletin of the Seismological Society of America, 88, 368-392.

Mallinson, I., P. Bharadwaj, G. Schuster, and H. Jakubowicz, 2011 , Enhanced refractor imaging by super-virtual interferometry: The Leading Edge, 30, 546, doi: 10.1190/1.3589113.

Mikesell, T. D., and K. van Wijk, 2011, Seismic refraction interferometry with a semblance analysis on the crosscorrelation gather: Geophysics, 76, no. 5, SA77-SA82, doi: 10.1190/geo2011-0079.1.

Mikesell, D., K. van Wijk, A. Calvert, and M. Haney, 2009, The virtual refraction: Useful spurious energy in seismic interferometry: Geophysics, 74, no. 3, A13-A17, doi: 10.1190/1.3095659.

Ruigrok, E., T. D. Mikesell, and K. van Wijk, 2012, Scanning for velocity anomalies in the crust and mantle with diffractions from the core-mantle boundary: Geophysical Research Letters, 39, L11301 (1-5), doi: 10.1029/ 2012GL051672.

Tatanova, M., K. Mehta, and B. Kashtan, 2009, Applications of virtual refraction in timelapse monitoring: 79th Annual International Meeting, SEG, Expanded Abstracts, 2617-2621.

Wapenaar, K., and J. Fokkema, 2006, Green's function representations for seismic interferometry: Geophysics, 71, no. 4, SI33-SI46, doi: 10.1190/1 .2213955 .

Yilmaz, Ö., 2001, Seismic data analysis: SEG. 